Canadian Oncology

Nursing Journal

Revue canadienne

de soins infirmiers

en oncologie

Volume 27, Issue 1 • Winter 2017

elSSN: $2368-8076$ 


\title{
Le sens de la vocation d'infirmière en oncologie : s'investir pour aider vraiment
}

\author{
par Lindsey Ann Davis, Frances Fothergill-Bourbonnais et Christine McPherson
}

\section{RÉSUMÉ}

La situation des soins prodigués aux personnes atteintes de cancer évolue. Les soins infirmiers en oncologie continuent à progresser et à répondre aux besoins évolutifs des patients atteints de cancer et de leur famille. Par contre, il $y$ a une compréhension limitée du sens véritable du travail d'infirmière en oncologie ainsi que des facteurs qui aident l'infirmière à faire son travail ou l'en empêchent. Dans le cadre de la présente étude, nous avons utilisé une approche phénoménologique interprétative. Six infirmières de deux unités de patients hospitalisés d'un établissement d'enseignement de soins tertiaires ont été interrogées. Le thème général S'investir pour aider vraiment reflète comment les infirmières en oncologie se sont investies pour établir une bonne relation avec les patients et leur famille et comment elles ont investi dans le développement de leurs connaissances et de leurs compétences jusqu'à façonner leur identité d'infirmières en oncologie. En retour, ces investissements ont nourri leur rôle et, par le soutien apporté tout au long de l'épreuve du cancer, sont venus changer la vie des patients et de leur famille. Les implications de ces constats pour les soins infirmiers en oncologie sont mises en évidence avec leurs relations avec la pratique, l'enseignement, la recherche et le leadership en soins infirmiers.

\section{INTRODUCTION}

T e portrait des soins contre le cancer continue d'évoluer et la Ltransition s'opère vers une plus grande offre de soins ambulatoires aux patients atteints de cancer. Les patients continuent néanmoins d'être soignés à l'hôpital pour les traitements chirurgicaux et, à certains moments, pour le diagnostic, la chimiothérapie, la gestion des symptômes et les soins palliatifs.

Comprendre le vécu des infirmières en oncologie est important pour la reconnaissance et le soutien de leur rôle

\section{AU SUJET DES AUTEURES}

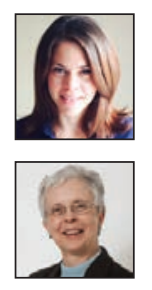

Auteur de correspondance : Lindsey Ann Davis, inf. aut., B.Sc.Inf., M.Sc.Inf.

\section{lindseyanndf@gmail.com}

Frances Fothergill-Bourbonnais, inf. aut., B.Sc.Inf., M.Inf., Ph. D., Professeure émérite, Université d'Ottawa, 451, chemin Smyth, RGN 3240, Ottawa, Ontario K1N 6N5

Téléphone : 613-562-5800, poste 8423; Télécopieur : 613-562-5443

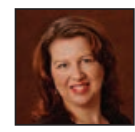

Christine McPherson, inf. aut., B.Sc., M.Sc., Ph. D., Professeure agrégée, Université d'Ottawa, 451, chemin Smyth, RGN 3251 D, Ottawa, Ontario K1N 6N5

Téléphone : 613-562-5800, poste 8394;

Télécopieur : 613-562-5443

DOI: $10.5737 / 236880762711521$ en matière de soins aux patients, étant donné qu'une cohorte d'infirmières spécialisées en oncologie est requise pour répondre aux besoins des patients cancéreux et de leur famille. Le Comité directeur des statistiques sur le cancer de la Société canadienne du cancer (2015) estime que près de la moitié de tous les Canadiens contracteront un cancer au cours de leur vie, et que le quart en mourra. Le nombre en hausse de nouveaux cas de cancer au Canada durant les 30 dernières années peut être attribuable à la croissance démographique et au vieillissement de la population, ainsi qu'à l'amélioration du dépistage et des traitements. Cette hausse devrait se poursuivre, car on prévoit que le nombre de Canadiens âgés de 65 ans et plus augmentera de plus du double, passant de 4,2 millions en 2003-2007 à 9,4 millions en 2028-2032. Nous aurons besoin d'infirmières spécialisées en oncologie pour soigner ces personnes.

Voici les questions qui ont guidé la présente recherche : quelle est l'expérience vécue d'une infirmière en oncologie dans une unité de soins hospitaliers, et quels sont les facteurs qui l'aident ou lui nuisent dans son travail?

\section{REVUE DE LA DOCUMENTATION}

Les soins infirmiers en oncologie sont exigeants à la fois sur les plans physique et émotionnel (Medland, HowardRuben et Whitaker, 2004). Les approches en matière de soins visent à satisfaire les besoins physiques, psychosociaux et spirituels des patients et de leur famille. De plus, les soins en oncologie sont orientés vers « le soulagement de la souffrance et le soutien en vue d'améliorer autant que possible la qualité de vie des patients qui font face à une maladie grave et souvent mortelle... » (Heidrich, 2007, p. 603).

La présence de conflits interdisciplinaires, les exigences $\mathrm{du}$ système, les restrictions en matière de soins, la lourdeur des cas et les besoins complexes de certains patients, l'impact émotionnel découlant de la grande implication auprès des patients et des familles, la confrontation avec la fin de vie et les questions éthiques sont autant de sources de stress pour les infirmières en oncologie (de Carvalho, Muller, de Carvalho et de Souza Melo, 2005; Grunfeld et al., 2000; Vachon, 2001). On compte toutefois peu de recherches sur les infirmières en oncologie. Ainsi, une recherche herméneutique menée par Rittman, Paige, Rivera, Sutphin et Godown (1997) a tenté de décrire l'expérience vécue par des infirmières en oncologie soignant des patients en fin de vie. Dans cette recherche, des entrevues approfondies avaient été effectuées auprès de six infirmières en oncologie qui comptaient au moins cinq années d'expérience de pratique avec des patients mourants. Quatre thèmes étaient abordés : 1) connaître le patient; 2) entretenir l'espoir; 3) faciliter la lutte; 4) protéger l'intimité 
et la vie privée. Les chercheurs sont arrivés à la conclusion que les infirmières composaient bien avec les exigences de leur travail sur le plan émotionnel en établissant divers degrés de proximité avec les patients à mesure qu'ils approchaient de la mort. Elles avaient décrit l'établissement de relations étroites avec certains patients et, même si elles n'établissaient pas ce type de liens avec tous les patients, elles sentaient qu'elles leur avaient fourni de bons soins à la fin de leur vie.

\section{MÉTHODOLOGIE}

Selon Steeves, Cohen et Wise (1994), l'approche phénoménologique peut procurer un « point de vue unique sur la nature des soins infirmiers en oncologie » (p. 19, traduction) parce que les infirmières ont la possibilité alors de réfléchir à leur expérience et au sens attribué à cette expérience. La phénoménologie s'intéresse à l'étude de l'être humain (Heidegger, 1962). L'approche phénoménologique interprétative de ce philosophe s'arrête à l'expérience de l'être humain dans sa vie quotidienne (Heidegger, 1962, p. 69). Selon lui, un chercheur peut uniquement arriver à établir la phénoménologie interprétative s'il a une connaissance antérieure du phénomène (McConnell-Henry, Chapman et Francis, 2009). La première auteure de l'étude a entamé sa démarche de recherche en partant d'hypothèses et de connaissances acquises dans le cadre de sa pratique comme infirmière en oncologie.

La phénoménologie interprétative utilisant l'approche de van Manen (1990) a servi de guide pour la présente étude, qui va au-delà d'une description de la manière dont un phénomène apparaît pour mieux dégager et comprendre le sens plus profond de l'expérience, exprimé verbalement et textuellement. Van Manen décrit la recherche phénoménologique herméneutique comme l'étude de l'expérience vécue d'une personne, sa réalité immédiate.

\section{DÉONTOLOGIE}

Nous avons obtenu l'approbation du comité de révision déontologique de l'organisation de soins de santé où l'étude s'est déroulée et de l'établissement de recherche universitaire. Des présentations ont été faites au personnel infirmier à l'établissement où le recrutement a eu lieu pour diffuser l'étude et inciter à y participer. Le consentement éclairé a été obtenu par écrit avant la tenue des entrevues.

\section{ÉCHANTILLONNAGE ET MILIEU}

Les infirmières en oncologie ont été recrutées par échantillonnage ciblé dans deux unités oncologiques d'un établissement d'enseignement de soins tertiaires à sites multiples de 1040 lits se trouvant dans un grand centre urbain de l'est de l'Ontario. Six infirmières âgées de 23 à 50 ans ont accepté d'être interrogées. Le nombre total d'années d'expérience en soins infirmiers oncologiques allait de 2 ans à plus de 30. Quatre des six infirmières avaient commencé leur carrière dans les unités sélectionnées pour l'étude. Les deux unités de soins infirmiers ont été conçues de manière à permettre la prestation de soins aux patients cancéreux à divers stades de la maladie.

Au moment de conduire l'étude, l'unité d'hémato-oncologie de l'hôpital comptait 23 lits pour patients atteints de leucémie aiguë. L'unité d'oncologie médicale totalisait quant à elle 35 lits pour patients présentant toutes sortes de cancers. Les soins étaient axés sur le diagnostic, la chimiothérapie, la prise en charge des symptômes et les soins palliatifs. Pour pouvoir participer à l'étude, les participantes devaient porter le titre d'infirmière autorisée et travailler dans au moins une des unités d'oncologie mentionnées, compter un minimum de deux années d'expérience professionnelle en oncologie, se considérer elles-mêmes comme infirmière en oncologie et bien parler anglais.

\section{COLLECTE DES DONNÉES}

Pour guider l'exploration de l'expérience vécue par les infirmières en oncologie, on a eu recours à des entrevues non structurées en seul à seul. Ces entretiens ont été menés par la première auteure. Des questions ouvertes comme «Quand avez-vous commencé à vous sentir réellement une infirmière en oncologie? » et «Qu'est-ce que ça signifie pour vous, être infirmière en oncologie? » ont été utilisées pour faire ressortir l'expérience vécue par les infirmières. Les entrevues, qui ont duré environ de 30 à 60 minutes, ont été enregistrées, puis transcrites.

\section{ANALYSE DES DONNÉES}

Dans la lignée de la phénoménologie heideggérienne, l'approche de van Manen (1990) a fourni un cadre d'analyse pour les entrevues transcrites et a permis de dégager la signification essentielle du phénomène. Van Manen a défini les thèmes d'une recherche phénoménologique comme les structures de l'expérience ayant contribué à comprendre un phénomène. Les données issues des transcriptions d'entrevues ont été analysées et guidées avec l'approche d'analyse thématique présentée par van Manen.

La structure essentielle du travail d'infirmière en oncologie a été décortiquée par un recours successif à une approche holistique, détaillée, puis sélective. La première auteure a d'abord lu les transcriptions au complet (approche holistique), puis ligne par ligne (approche détaillée). Ce procédé a permis de s'imprégner complètement des données. Les pensées et mots fréquents des participantes ont été identifiés et codés à l'aide de couleurs pendant la lecture de chaque entrevue. Les phrases importantes ont ensuite été compilées pour former les sous-thèmes, qui ont eux-mêmes été regroupés en thèmes. Les relations entre les thèmes ont été examinées au sein de chacune des entrevues et entre celles-ci. Les grands thèmes ont ainsi été dégagés, par l'observation et l'interprétation de la signification et du contexte propres au récit livré par chaque participante, et par le processus d'écriture et de réécriture. Les chercheurs ont régulièrement reconsulté les transcriptions à mesure que le procédé d'écriture progressait pour situer les thèmes dans les phrases et les renseignements communiqués par les participantes (approche sélective). Tous les chercheurs ont examiné les transcriptions et atteint un consensus quant aux thèmes à retenir. Si le consensus n'était pas atteint entre les chercheurs, ceux-ci retournaient aux données. 


\section{MÉTHODES POUR ASSURER LA RIGUEUR SCIENTIFIQUE}

Les quatre critères de rigueur scientifique de Lincoln et Guba (1985), à savoir la crédibilité, la fiabilité, la constance interne et la transférabilité, ont été appliqués. La crédibilité a été respectée en retournant voir les participantes pour leur demander de vérifier le résumé des thèmes et des catégories et en obtenant l'accord de tous les chercheurs sur les thèmes développés. La crédibilité a été également renforcée du fait que deux des chercheurs (Frances Fothergill-Bourbonnais et Christine McPherson) étaient des expertes des méthodes qualitatives. On a satisfait au critère de fiabilité par la tenue de discussions entre les chercheurs tout au long du processus d'analyse, ainsi que pendant l'élaboration et l'utilisation d'une piste de vérification devant servir à expliquer et à rendre transparentes toutes les décisions prises par les chercheurs. Pour le critère de constance interne, un journal de bord documentant les croyances et les hypothèses de la première auteure a été tenu tout au long de la recherche. Enfin, la transférabilité des constats posés a été obtenue et mise en lumière grâce à la description détaillée des thèmes, des participantes et du contexte entourant leurs soins.

\section{CONSTATS}

Le thème général, S'investir pour aider vraiment, décrit ce que la vocation d'infirmière en oncologie signifie. Ce thème général se décompose en quatre thèmes : Soigner la personne dans toutes ses dimensions, Défendre les intérêts du patient, Trouver le juste équilibre et Avoir le sentiment de faire partie de quelque chose de bien. Les thèmes et les catégories associées figurent au figure 1 .

\section{S'investir pour aider vraiment}

Les participantes ont dit s'investir dans la vie des patients, dans leur travail d'infirmière, et auprès de leurs collègues. Elles s'engagent à accompagner les patients et leur famille pendant qu'ils vivent les impacts du cancer. En même temps, elles sont témoins de la souffrance des patients et des familles, et essaient de tisser des liens de manières qui leur paraissent significatives. Les participantes reconnaissaient investir aussi en ellesmêmes pour s'assurer d'avoir le bagage requis de connaissances et de compétences cliniques pour répondre aux besoins de plus en plus spécialisés des patients en oncologie. Enfin, une fois qu'elles se sentaient bien intégrées dans une équipe, elles disaient pouvoir alors redonner aux autres infirmières.

\section{Soigner la personne dans toutes ses dimensions}

Le thème Soigner la personne dans toutes ses dimensions vient refléter la nature holistique des soins infirmiers en oncologie. Il rassemble trois catégories : Avoir une vue d'ensemble, Partager des moments avec les patients, et Rester présent tout au long de l'épreuve. Les participantes ont souligné la façon dont elles en sont venues à connaître de près les personnes qu'elles soignaient pendant des maladies parfois très longues. L'une d'entre elles l'a décrit ainsi : « Nous nous occupons de la personne entière, pas juste de son corps... On doit toujours considérer le patient dans toutes ses dimensions. »

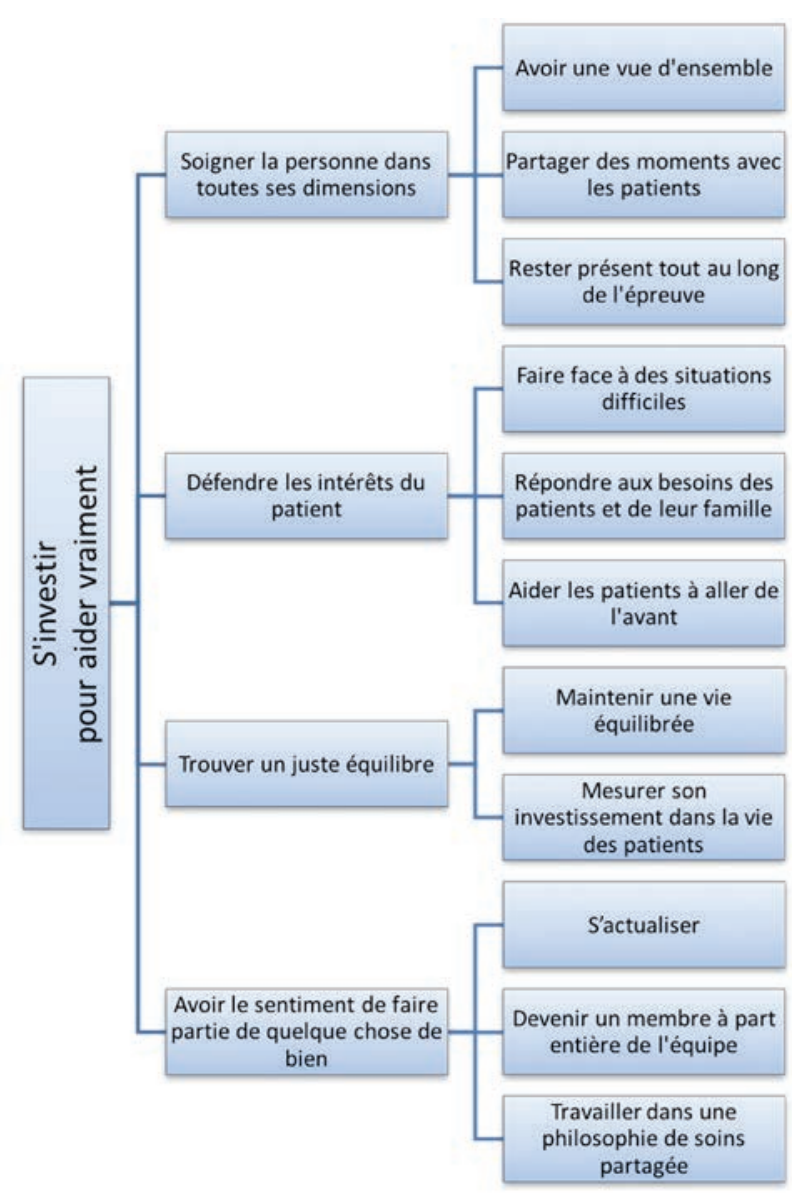

Figure 1.

Avoir une vue d'ensemble. Avoir une vue d'ensemble procure aux infirmières un moyen pour comprendre le vécu de leurs patients autant entre les murs de l'hôpital qu'à l'extérieur. Les participantes ont décrit comment elles étaient témoins des nombreux effets du cancer sur les plans psychosocial, physique et spirituel de la vie des patients. « [...] Le cancer a des répercussions sur toutes les sphères de la vie $[. .$.$] », expliquait$ l'une d'entre elles. Cette connaissance des patients oriente les soins prodigués par les infirmières, comme l'illustre cette réponse : « [...] C'est comme si on vérifiait tous leurs morceaux [du patient] en s'assurant que tout va fonctionner pour eux lorsqu'ils obtiendront leur congé de l'hôpital. »

Partager des moments avec les patients. Les infirmières passent du temps avec les patients et partagent donc des moments avec eux. Dire un bonjour en passant ou passer quelques minutes à regarder une partie de hockey avec un patient sont des gestes simples qui étaient gratifiants et procuraient de la satisfaction aux participantes. Ainsi témoignait une participante qui avait réconforté un patient en pleine nuit : « [...] lorsqu’une personne n'arrive pas à dormir parce qu'elle se demande comment la journée se passera et qu'on passe un moment avec elle, ça, ça compte. »

Rester présent tout au long de l'épreuve. Le parcours que les participantes partagent avec leurs patients se déroule au fil du temps, « [...] en partie parce que nos patients partent et 
reviennent tout au long du traitement, et ce, sur une période d'au moins un an », comme le mentionnait une participante. Il est fréquent pour des patients en oncologie et leur famille d'avoir à subir de nombreuses et longues hospitalisations. Toutes les réponses des participantes ont montré un engagement à demeurer présentes au fil du temps, à être avec leurs patients pendant toutes leurs hospitalisations. Les participantes ont considéré cela comme un privilège et une manière pour les patients d'établir une relation fondée sur la confiance avec les participantes.

\section{Défendre les intérêts du patient}

Le thème Défendre les intérêts du patient fait ressortir les défis des infirmières lorsqu'elles prennent soin des patients et de leur famille de même que la persévérance dont elles font preuve pour répondre aux besoins. Ce thème regroupe trois catégories : Faire face à des situations difficiles, Répondre aux besoins des patients et de leur famille et Aider les patients à aller de l'avant. Ce thème présente les manières dont les infirmières soutiennent les patients cancéreux dans leur épreuve et les difficultés auxquelles les infirmières en oncologie sont confrontées en donnant ce soutien.

Faire face à des situations difficiles. Parmi les situations décrites par les participantes, on compte celle où il faut soigner des patients dans l'attente de leur pronostic ou diagnostic. «On sait pourquoi ils sont dans l'unité... Le patient attend là tout le weekend jusqu'à apprendre qu'il a la leucémie et commencera un traitement, mais personne n'a pris les devants pour leur parler de leur biopsie de la moelle épinière [...] » Les visions incongrues quant au plan de soins entre les patients et leur famille et les professionnels de la santé ont aussi été présentées comme une source de difficultés, comme le relate une infirmière : «Cette personne a des demandes concernant son traitement qui ne lui apporteront rien du tout, alors c'est difficile... On s'inquiète de la manière dont les choses tourneront pour elle. » En dépit de ces défis, les participantes se concentrent sur la prestation des soins aux patients et le soutien à leur famille. «On doit être solide et aimer son travail... Parce que même si on aide cette personne, ce ne sera pas facile. »

Répondre aux besoins des patients et de leur famille. Les participantes ont expliqué répondre aux besoins des patients en les aidant à avoir une compréhension réaliste de leur cancer. Pour comprendre à fond les besoins des patients et de leur famille, les participantes ont passé du temps avec eux à discuter et leur poser des questions. L'accumulation des interactions au fil des hospitalisations aide à comprendre le vécu des patients et leurs besoins. « Parfois, les croyances des gens viennent de ce qu'ils voient à la télé ou encore de ce qu'a vécu leur grand-mère il y a 50 ans... Une partie du rôle de l'infirmière en oncologie est de leur donner l'heure juste et de les aider à comprendre le système d'aujourd'hui. »

Aider les patients à aller de l'avant. Par leurs interactions avec les patients, les participantes en sont venues à les connaître, de même que leurs désirs et objectifs. "C'est formidable parce que c'est pour ça que je le fais... et si ça signifie qu'ils peuvent fonctionner ou aller de l'avant, alors c'est important. »
Une participante a raconté être très émue lorsque sa patiente a obtenu son congé de l'hôpital après avoir composé avec de nombreux défis tout au long son hospitalisation.

\section{Trouver un juste équilibre}

Le thème Trouver un juste équilibre reflète la nature émotionnelle des soins infirmiers en oncologie et l'équilibre nécessaire pour gérer ces expériences. Le thème chapeaute deux catégories : Maintenir une vie équilibrée et Mesurer son investissement dans la vie des patients.

Maintenir une vie équilibrée. Les participantes ont évoqué l'équilibre à maintenir entre leur vie professionnelle et leur vie personnelle en recourant notamment à l'analogie d'un funambule tenant un balancier pour illustrer leur expérience. Si le balancier commence à pencher trop d'un côté, le funambule est déséquilibré. Pour ces infirmières en oncologie, l'équilibre du balancier entre leur travail et leur vie à elles était bel et bien au rendez-vous. Dans ce jeu d'équilibre, si une situation au travail crée de l'instabilité, le fait de s'en confier avec d'autres infirmières par un partage d'expérience aide à retrouver son ballant et à ne pas tomber. Comme le disait une participante : « [C'est bon de] savoir que les autres infirmières en oncologie comprennent ce que je vis... parce que les gens qui ne font pas le même travail que nous ne comprennent pas toujours, eux. »

Mesurer son investissement dans la vie des patients. Pour les soigner, les participantes s'investissent auprès des patients et de leur famille. Au fil du temps, elles apprennent à mieux connaître les patients et les membres de leur famille et établissent des relations étroites au gré des hospitalisations répétitives. Cependant, les participantes demeurent vigilantes quant à leur engagement et leur investissement émotionnel en cherchant à le gérer. Selon une infirmière : « Si on devient trop subjectives, on n'est pas fonctionnelles dans notre rôle, on devient trop impliquée dans la situation. » En gérant le niveau d'investissement émotionnel, les participantes peuvent continuer d'agir de manière professionnelle.

\section{Avoir le sentiment de faire partie de quelque chose de bien}

Le dernier thème, Avoir le sentiment de faire partie de quelque chose de bien, traduit l'expérience des infirmières en tant que membres d'une équipe dévouée à l'atteinte d'objectifs communs. Le thème englobe trois catégories : S'actualiser, Devenir un membre à part entière de l'équipe et Travailler dans une philosophie de soins partagée.

S'actualiser. Les participantes ont énuméré les types de connaissances et de compétences nécessaires pour bien fonctionner dans leur rôle d'infirmières en oncologie. Les connaissances sur le cancer et les approches de traitement évoluent sans cesse, ce qui nécessite une mise à jour continuelle pour s'assurer d'avoir des connaissances à jour et exactes, notamment sur les manifestations pathophysiologiques du cancer, les paramètres biologiques, les complications associées et les traitements comme la chimiothérapie. Même si « s'actualiser » a été désigné comme un grand défi, ces connaissances sont indispensables pour améliorer la pratique des soins infirmiers. Cette réflexion d'une participante l'illustre : 
« Le plus grand défi est de travailler avec les nouveaux médicaments liés au domaine, les nouvelles chimiothérapies, et d'essayer de suivre cela de près... Il y a toujours des façons d'améliorer notre pratique en soins infirmiers ».

Devenir un membre à part entière de l'équipe. Le perfectionnement et le développement des infirmières en oncologie sont venus, au fil du temps, consolider leur expertise en oncologie à mesure qu'elles gagnaient en confiance et en aisance dans leur rôle en tant que membre utile à l'équipe. En s'investissant dans leur travail, les participantes sont devenues plus confiantes dans leur rôle d'infirmières en oncologie. Un délai d'au moins un an devait passer avant que les participantes commencent à se considérer elles-mêmes comme des infirmières en oncologie. En gagnant en confiance, les participantes ont décrit qu'elles se sentaient ensuite en mesure de « redonner », de soutenir les autres membres de l'équipe. Une infirmière se rappelait sa rencontre avec une infirmière moins expérimentée : « Je n'avais pas l'habitude qu'on me demande des choses... Alors c'était difficile, et puis j'ai constaté que j'avais de l'expérience, et que je savais ce que je faisais, je me suis donc ensuite sentie comme une membre à part entière de l'équipe... »

Travailler dans une philosophie de soins partagée. Cette catégorie reflète l'expérience vécue par les participantes comme membres d'équipes des soins infirmiers et d'équipes interprofessionnelles. Elles ont décrit comment les membres font équipe pour offrir des soins dans un but commun : aider le patient. Tout en étant interrogées sur leur rôle propre, les infirmières en oncologie reconnaissaient faire aussi partie d'une équipe. Comme le faisait remarquer l'une des participantes : « Nous ne sommes pas seules avec le patient... nous faisons partie d'un groupe plus vaste de personnes qui leur prodiguent des soins. »

\section{DISCUSSION}

Les constats de cette étude font ressortir que les infirmières en oncologie évoluant dans le contexte étudié voient leurs patients de manière holistique et que cette attitude oriente leurs soins. Les infirmières reconnaissaient que le cancer vient affecter chaque aspect de la vie de leurs patients et de leur famille. La conceptualisation de l'holisme en soins infirmiers, telle que décrite par Clark (2012), représente le patient comme un être multidimensionnel en reconnaissant que la perturbation de l'une de ses dimensions (p. ex. psychosociale, physique, émotionnelle...) peut venir affecter les autres dimensions qui le composent.

Cette vision holistique est au cœur de la manière dont les infirmières participant à la présente étude sont parvenues à connaître chaque patient et à saisir leurs souhaits et leurs besoins. Des relations se sont tissées et ont été entretenues au fil des interactions dans le temps, parfois sur plusieurs années. C’est grâce à ces relations que la confiance, la présence réconfortante et la relation thérapeutique sont maintenues (Carter, 2009). Une relation infirmière-patient peut être décrite comme une relation d'aide fondée sur la confiance et le respect mutuels, l'entretien de l'espoir, l'écoute de soi et des autres, et l'aide pour satisfaire les besoins physiques, émotionnels et spirituels en s'appuyant sur ses connaissances et compétences (Carter, 2009). En prenant soin des patients et de leur famille, les infirmières en oncologie peuvent utiliser leur présence physique pour apporter chaleur, réconfort et humanité et ainsi soulager la souffrance qui peut accompagner le cancer (Ferrell et Coyle, 2008).

Connaître leurs patients et leurs soins signifiait pour ces infirmières d'être plus aptes à répondre à leurs besoins et à défendre leurs droits. Apprendre à connaître le patient, comme l'indiquent Tanner, Benner, Chesla et Gordon (1993), c'est le connaître non seulement en sachant comment il risque de réagir, mais aussi en sachant qui il est en tant que personne. La défense des droits des patients, comme l'ont décrit $\mathrm{Bu}$ et Jezewski (2007), est « un processus ou une stratégie qui consiste en une série de mesures précises visant à préserver, à représenter ou à protéger les droits des patients, leur meilleur intérêt et leurs valeurs dans le système de soins de santé » (p. 104, traduction). Les travaux de recherche examinant la défense des droits des patients dans le domaine des soins infirmiers en oncologie ont relevé trois activités globales de protection : l'analyse, le conseil et l'intervention (VaartioRajalin et Leino-Kilpi, 2011). Dans cette étude, la défense des droits est ressortie comme un rôle clé en matière de soins infirmiers du fait que les infirmières sont plus en contact que tout autre membre de l'équipe soignante avec les patients.

La nature émotionnelle des défis rencontrés comme infirmière en oncologie et les investissements consentis se faisaient jour au fur et à mesure des récits des participantes. Les infirmières estimaient acquérir un rôle significatif dans la vie de leurs patients au fil du temps, en les accompagnant dans cette épreuve de maladie. Cet investissement émotionnel et le niveau d'engagement auprès des patients et de leur famille constitue en outre un exercice d'équilibre, un constat appuyé par Rittman et collaborateurs (1997). Boyle (2000) mentionne que l'on doit reconnaître les aspects psychologiques et émotionnels dans les fonctions de l'infirmière en oncologie, et que son importance doit faire la paire avec des compétences techniques poussées. Dans la présente étude, cette reconnaissance et le sentiment de faire partie de l'équipe de soins infirmiers se sont construits au fil du temps, à mesure que les infirmières en oncologie rencontrées ont vu s'accroître leurs compétences et leur confiance. Les collègues infirmières ont offert du mentorat et se sont soutenues mutuellement pour résoudre les situations cliniques complexes des patients. Elles se sont de surcroît soutenues dans leur travail pour mieux en gérer les aspects émotionnels. Selon Haavardsholm et Naden (2009), le « réseau professionnel » aide l'infirmière à avoir confiance en elle lorsque ce réseau de collègues lui apporte du soutien. Les lignes directrices des pratiques exemplaires des milieux de travail sains (Association des infirmières et infirmiers autorisés de l'Ontario, 2006) nomment l'échange d'expertise parmi les éléments encourageant l'esprit d'équipe. Lorsque l'infirmière a un sentiment d'appartenance, elle est aussi plus susceptible de rester en poste (Tourangeau, Cummings, 
Cranley, Ferron et Harvey, 2010). La transition pour devenir un membre à part entière de l'équipe de soins infirmiers coïncide avec la prise de conscience qu'à titre d'infirmières, elles sentent qu'elles peuvent répondre aux besoins de leurs patients et aux exigences de leurs fonctions. Dans une étude portant sur la première année en soins infirmiers d'infirmières en oncologie pédiatrique, Linder (2009) a constaté qu'en plus de gagner en confiance et en compétences, les participantes se considéraient comme des membres légitimes de l'équipe, tout comme les participantes dans la présente étude.Le temps a été un facteur étroitement lié à tous les thèmes. En effet, il faut du temps pour s'investir et ainsi arriver à aider vraiment. Puisque les patients revenaient souvent maintes fois à l'hôpital au fil des ans pour recevoir traitements et soins, le temps devient un élément clé pour établir et développer des relations avec les patients et leur famille. Avec le temps, les infirmières en sont venues à connaître leurs patients, ce qui les a aidées à mieux comprendre leurs besoins et à défendre leurs droits. Répondre aux besoins multiples des patients et de leur famille dans toute leur complexité prend forcément du temps. Du temps a aussi été investi par les participantes pour devenir compétentes sur le plan clinique et, grâce à cet investissement, elles ont gagné en compétence et en expérience. C'est le temps qui, essentiellement, a aidé les infirmières à considérer qu'elles étaient finalement devenues des infirmières en oncologie.

\section{IMPLICATIONS POUR LES SOINS INFIRMIERS EN ONCOLOGIE}

Les constats de la présente étude aident à comprendre à quoi correspond la profession d'infirmière en oncologie, et cela a des effets sur la pratique, la formation, la recherche et le leadership. Le temps a été un élément clé dans l'établissement et le développement des relations avec les patients et leur famille. On doit donner aux infirmières la possibilité de développer de bonnes relations par leurs interactions avec les patients et leur famille, et pour ce faire, elles ont besoin de temps. Les chefs des soins infirmiers doivent reconnaître que ce temps est nécessaire à un bon soutien des patients et de leur famille, et que les interventions en soins infirmiers y contribuent.

Dans la présente étude, le temps investi faisait une différence. Perry (2008) a décrit l'expérience des infirmières en oncologie comme le fait d'avoir des moments de connexion et de faire en sorte que ces moments comptent. Les infirmières cheminent avec les patients, construisant au fil $\mathrm{du}$ temps une relation avec eux, notamment en raison des nombreuses hospitalisations requises. Leur présence aide les patients à faire ce chemin et à vivre leur transition vers la guérison ou la fin de la vie. Corner (1997) a insisté sur le fait que ce travail de cheminement des infirmières auprès des patients devait être reconnu.Le sens que les infirmières attribuent à leur expérience est étroitement lié au contexte des milieux où elles travaillent. Comme les infirmières répondent aux besoins des patients dans ces milieux, le sens qu'elles donnent à leur travail est imbriqué dans leurs interactions avec les patients (Edvardsson, Sandman et Rasmussen, 2006). Lorsqu'un milieu de soins infirmiers procure du soutien, il est plus probable que les infirmières resteront en poste et qu'elles continueront de s'investir dans leur milieu de travail. L'étude fait ressortir l'importance que les infirmières attachent au soutien les unes des autres, car celui-ci est lié à l'atteinte d'un bien-être émotionnel, à la confiance, ainsi qu'aux connaissances, au jugement et à l'acquisition de compétences. De plus, lorsque les infirmières se sentent appuyées et qu'elles font partie de l'équipe interprofessionnelle, elles peuvent s'investir encore plus et donner en retour à l'équipe et à l'unité des soins infirmiers. Cohen, Ferrell, Vrabel, Visovski et Schaefer (2010) indiquent que les infirmières en oncologie ont besoin d'être soutenues pour pouvoir faire leur travail.

Le travail d'infirmière en oncologie dans la présente étude a été lié à l'investissement fait dans le développement des connaissances et des compétences, ce qui inclut d'être à jour par rapport aux méthodes de traitement et aux pratiques de soins infirmiers. Les connaissances et les compétences peuvent s'acquérir et se raffiner par les expériences personnelles et professionnelles (Cohen, 1995; Kendall, 2006). Les hôpitaux peuvent s'associer à une organisation pour obtenir de la formation et du mentorat afin de favoriser l'acquisition des connaissances et le développement des soins infirmiers par le biais d'initiatives de mentorat.

\section{CONCLUSION}

Les constats de la présente étude aident à comprendre ce que la vocation d'infirmière en oncologie signifie. Les infirmières en oncologie s'investissent dans leurs vies personnelle et professionnelle afin d'aider vraiment les patients et leur famille lorsqu'ils sont confrontés au cancer. Le travail d'infirmière en oncologie nécessite du temps : du temps à passer avec les patients, du temps pour tisser des liens avec les patients et leur famille, du temps pour réfléchir à son expérience et échanger avec les autres. Les infirmières en oncologie ont aussi besoin de temps pour continuer à acquérir des connaissances et des compétences cliniques qui leur permettront de prodiguer des soins ciblés répondant aux besoins de leurs patients.

Les infirmières en oncologie s'engagent dans l'acquisition de leurs propres connaissances et compétences, ainsi que dans le soutien du développement de leurs collègues. Ce faisant, elles deviennent des membres à part entière de leur équipe, à laquelle elles redonnant, pouvant ainsi donner et recevoir du soutien au sein de la structure de l'unité de soins infirmiers. Cette structure privilégiant un milieu de travail favorable à l'entraide entre les membres d'une équipe peut soutenir la capacité des infirmières en oncologie à continuer d'aider vraiment et à ainsi faire une différence dans la vie des autres.

\section{REMERCIEMENTS}

Cynthia Toman et Inara Karrei pour leur soutien apporté dans le cadre de la présente recherche. 


\section{RÉFÉRENCES}

Association des infirmières et infirmiers autorisés de l'Ontario / Registered Nurses' Association of Ontario (RNAO). (2006). Collaborative practice among nursing teams. Consulté sur http:// rnao.ca/sites/rnaoca/files/Collaborative_Practice_Among_ Nursing_Teams.pdf

Boyle, D. (2000). Pathos in practice: Exploring the affective domain of oncology nursing. Oncology Nursing Forum, 27, 915-919.

$\mathrm{Bu}, \mathrm{X}$. et Jezewski, M. (2007). Developing a mid-range theory of patient advocacy through concept analysis. Journal of Advanced Nursing, 57(1), 101-110.

Carter, M. (2009). Trust, power, and vulnerability: A discourse on helping in nursing. Nursing Clinics of North America, 44(4), 393-405.

Clark, C. (2012). Beyond holism: Incorporating an integral approach to support caring-healing-sustainable nursing practices. Holistic Nursing Practice, 26(2), 92-102.

Cohen, M.Z. (1995). The meaning of cancer and oncology nursing: Link to effective care. Seminars in Oncology Nursing, 11(1), 59-67.

Cohen, M.Z., Ferrell, B.R., Vrabel, M., Visovsky, C. et Schaefer, B. (2010). What does it mean to be an oncology nurse? Re-examining the life cycle concepts. Oncology Nursing Forum, 17(5), 561-570.

Comité directeur des statistiques sur le cancer de la Société canadienne du cancer (2015). Statistiques canadiennes sur le cancer 2015 : Prévisions concernant le fardeau futur du cancer au Canada. Consulté sur http://www.cancer.ca/ /media/cancer. ca/CW/cancer\%20information/cancer\%20101/Canadian\%20 cancer\%20statistics / Canadian-Cancer-Statistics-2015-FR. pdf?la=fr-CA

Corner, J. (1997). Beyond survival rates and side effects: Cancer nursing as therapy. Cancer Nursing, 20(1), 3-11.

de Carvalho, E.C., Muller, M., de Carvalho, P.B. et de Souza Melo, A. (2005). Stress and the professional practice of oncology nurses. Cancer Nursing, 28(3), 187-192.

Edvardsson, D., Sandman, P. et Rasmussen, B. (2006). Caring or uncaring-Meanings of being in an oncology environment. Journal of Advance Nursing, 55(2), 188-197.

Ferrell, B. et Coyle, N. (2008). The nature of suffering and the goals of nursing. Oncology Nursing Forum, 35(2), 241-247.

Grunfeld, E., Whelan, T., Zitzelsberger, L., Willan, A., Montesanto, B. et Evans, W. (2000). Cancer care workers in Ontario: Prevalence of burnout, job stress and job satisfaction. Canadian Medical Association Journal, 163, 166-169.

Haavardsholm, I. et Naden, D. (2009). The concept of confidenceThe nurse's perception. European Journal of Cancer Care, 18, 483-491.
Heidegger, M. (1962). Being and time (J. MacQuarrie et E. Robinson, traducteurs). New York: Harper \& Row Publishers (œuvre originale publiée en 1927 sous le titre Sein und Zeit, Gesamtausgabe, 2).

Heidrich, D. (2007). Palliative care. Dans M.E. Langhorne, J.S. Fulton et S.E. Otto (éditeurs). Oncology Nursing (5th ed.). St. Louis : Elsevier. 602-619.

Kendall, S. (2006). Admiring courage: Nurses' perceptions of caring for patients with cancer. European Journal of Oncology Nursing, 10(5), 324-334.

Lincoln, Y. et Guba, E. (1985). Naturalistic inquiry. Beverly Hills : Sage Publications.

Linder, L. (2009). Experiences of pediatric oncology nurses: The first year of hire. Journal of Pediatric Oncology Nursing, 26(1), 29-40.

McConnell-Henry, T., Chapman, Y. et Francis, K. (2009). Husserl and Heidegger: Exploring the disparity. International Journal of Nursing Practice, 15, 7-15.

Medland, J., Howard-Ruben, J. et Whitaker, E. (2004). Fostering psychosocial wellness in oncology nurses: Addressing burnout and social support in the workplace. Oncology Nursing Forum, 31(1), 47-54.

Perry, B. (2008). Pourquoi les infirmières modèles en oncologie semblent-elles éviter l'usure de compassion? Revue canadienne de soins infirmiers en oncologie, 18(2), 93-99.

Rittman, M., Paige, P., Rivera, J., Sutphin, L. et Godown, I. (1997). phenomenological study of nurses caring for dying patients. Cancer Nursing, 20(2), 115-119.

Steeves, R., Cohen, M.Z. et Wise, C. (1994). An analysis of critical incidents describing the essence of oncology nursing. Oncology Nursing Forum, 21(8 Suppl.), 9-17.

Tanner, C.A., Benner, P., Chesla, C. et Gordon, D.R. (1993). The Phenomenology of knowing the patient. Journal of Nursing Scholarship, 25(4), 273-280.

Tourangeau, A., Cummings, G., Cranley, L., Ferron, E. et Harvey, S. (2010) Determinants of hospital nurse intention to remain employed: Broadening our understanding. Journal of Advanced Nursing, 66(1), 22-32.

Vaartio-Rajalin, H. et Leino-Kilpi, H. (2011) Nurses as patient advocates in oncology care: Activities based on literature. Clinical Journal of Oncology Nursing, 15(5), 526-532.

Vachon, M.L.S. (2001). The nurses' role: The world of palliative care nursing. In B.R. Ferrell \& N. Coyle (éditeurs). Textbook of palliative nursing (647-662). New York: Oxford University Press.

van Manen, M. (1990). Researching lived experience: Human science for an action sensitive pedagogy. London, Ontario, Canada : Althouse Press. 